\title{
Reducing carrier escape in the InAs/GaAs quantum dot intermediate band solar cell
}

\author{
E. Antolín, ${ }^{1, a)}$ A. Marti, ${ }^{1}$ C. D. Farmer, ${ }^{2}$ P. G. Linares, ${ }^{1}$ E. Hernández, ${ }^{1}$ A. M. Sánchez, ${ }^{3,4}$ \\ T. Ben, ${ }^{3}$ S. I. Molina, ${ }^{3}$ C. R. Stanley, ${ }^{2}$ and A. Luque ${ }^{1}$ \\ ${ }^{1}$ Instituto de Energía Solar, E.T.S.I. Telecomunicación. Universidad Politecnica de Madrid, 28040 Madrid. \\ Spain \\ ${ }^{2}$ Department of Electronics and Electrical Engineering, University of Glasgow, Glasgow $G 128 Q Q$. \\ United Kingdom \\ ${ }^{3}$ Dpto. de Ciencia de los Materiales e Ingeniería Metalírgica y Quimica Inorganica. Facultad de Ciencias. \\ Universidad de Cadiz, 11510 Puerto Real, Cadiz, Spain \\ ${ }^{4}$ Department of Physics. University of Warwick, Wanwick CV4 7AL. United Kingdom
}

\begin{abstract}
Intermediate band solar cells (IBSCs) fabricated to date from $\operatorname{In}(\mathrm{Ga}) \mathrm{As} / \mathrm{GaAs}$ quantum dot arrays (QD-IBSC) exhibit a quantum efficiency (QE) that extends to below bandgap energies. However, the production of sub-bandgap photocurrent relies often on the thermal and/or tunneling escape of carriers from the QDs, which is incompatible with preservation of the output voltage. In this work, we test the effectiveness of introducing a thick GaAs spacer in addition to an InAlGaAs strain relief layer (SRL) over the QDs to reduce carrier escape. From an analysis of the QE at different temperatures, it is concluded that escape via tunneling can be completely blocked under short-circuit conditions, and that carriers confined in QDs with an InAlGaAs SRL exhibit a thermal escape activation energy over $100 \mathrm{meV}$ larger than in the case of InAs QDs capped only with GaAs.
\end{abstract}

\section{INTRODUCTION}

The intermediate band solar cell (IBSC) (Ref. 1) is a novel photovoltaic device with a higher efficiency limit than conventional (single-gap) solar cells [63.2\% (Ref. 1) versus $40.7 \%,{ }^{2,3}$ assuming maximum sunlight concentration in both cases]. One approach that is being followed to implement the IBSC is the use of quantum dots (QDs), ${ }^{4}$ most frequently In(Ga)As/GaAs QD arrays. ${ }^{5-11}$ The QD-IBSC prototypes fabricated with this system have served to demonstrate the operation principles of the IBSC model ${ }^{12,13}$ but they still suffer from parasitic loss mechanisms. In particular, it has been found that the respective electronic populations of the intermediate band (IB) and the conduction band (CB) cannot be split sufficiently. ${ }^{14}$ As a result, in the $\operatorname{In}(\mathrm{Ga}) \mathrm{As} / \mathrm{GaAs}$ QDIBSCs demonstrated to date the sub-bandgap (SBG) IB-CB optical transition is replaced by carrier escape mechanisms. In this work we present and evaluate a new QD-IBSC structure where carrier escape from the IB to the $\mathrm{CB}$ is reduced. We demonstrate that two aspects of the QD-IBSC design have to be modified to suppress this carrier escape. First, the carrier escape in QD-IBSCs has a strong thermal component. This component has been reduced in our samples by introducing an InAlGaAs strain relief layer (SRL) over the QDs, which changes the band structure and increases the activation energy of confined carriers. Second, in the typical QD-IBSC structure, an additional IB-CB escape component arises from tunneling of confined carriers to the barrier, for which carriers can use the QD levels of adjacent layers as intermediate steps. In the new QD-IBSC structure presented here,

"Electronic mail: elisa@ies-def.upn.es. the tunneling mechanism has been blocked owing to the increased thickness of the GaAs spacers that separate the QD layers.

Strong carrier escape between IB and CB has to be avoided because it is incompatible with preservation of the output voltage and the realization of the high efficiencies predicted by the IBSC theoretical model. To illustrate this point, we will review briefly the IBSC theory in Sec. I. Detailed descriptions of the IBSC model can be found in earlier publications. ${ }^{1,15,16}$ Section II will describe the samples used in the experiments, their rationale and manufacture. The experimental results will be presented in Sec. III and will be discussed in terms of the carrier escape activation energy.

\section{BASIC IBSC MODEL AND IMPLEMENTATION WITH QDS}

As depicted in the band diagram of Fig. 1(a), in an IBSC the absorbing material (IB material) exhibits an isolated energy band, the IB, within the fundamental gap $\left(E_{\mathrm{G}}\right)$; this band divides $E_{\mathrm{G}}$ into two SBGs, $E_{\mathrm{L}}$ and $E_{\mathrm{H}}$. An electronhole pair can be generated in this material by two mechanisms: absorption of one photon in the transition labeled (3) or absorption of two SBG photons through transitions (1) and (2). To avoid a reduction in the output voltage, it is necessary that the electronic populations associated with the IB, the CB and the valence band (VB) are each described by its owl quasi-Fermi level (denoted $\varepsilon_{\mathrm{FB}}$ for carriers in the IB, $\varepsilon_{\mathrm{Fe}}$ for electrons in the $\mathrm{CB}$, and $\varepsilon_{\mathrm{Fh}}$ for holes in the $\mathrm{VB}$ ). This means that carrier relaxation within the bands (thermalization) is a much faster process than carrier relaxation between bands (recombination). Also, two layers of conventional semiconductor of opposite doping ( $p$-emitter and $n$-emitter) 
(a)
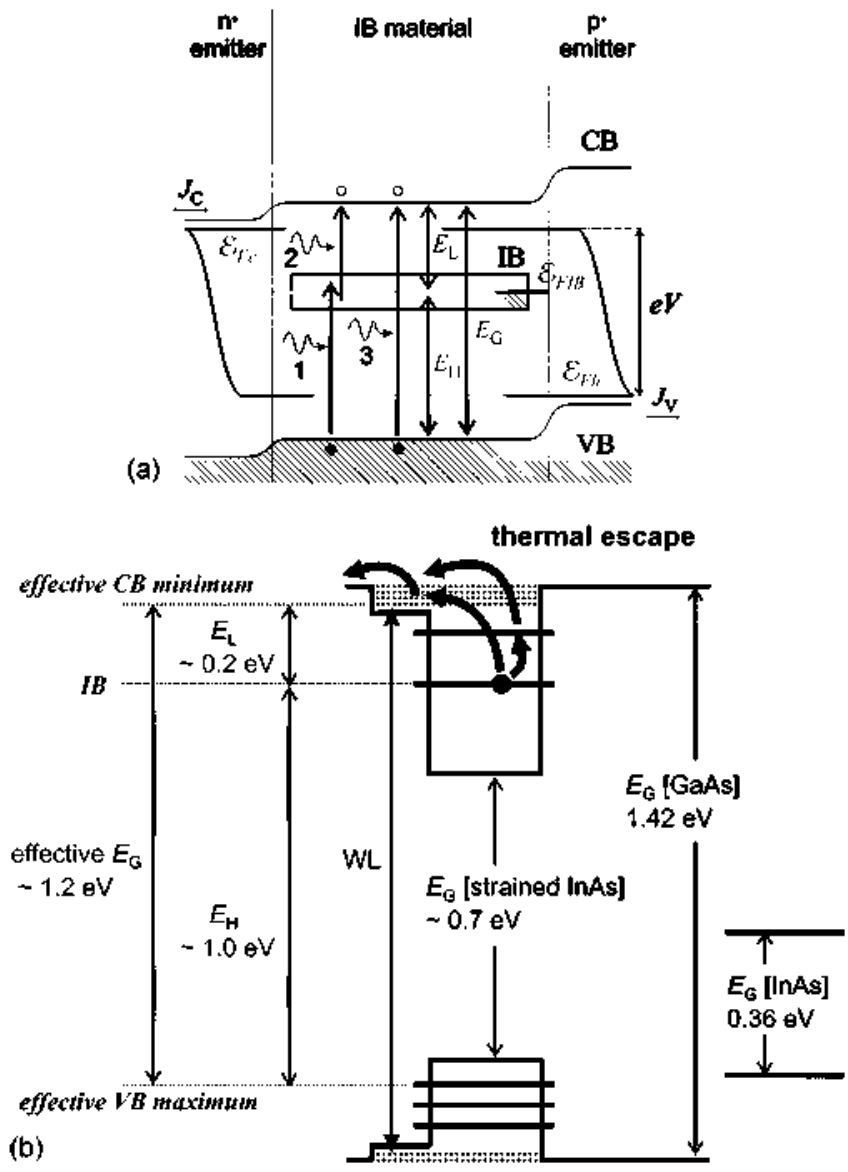

FIG. 1. (Colot online) (a) Band diagram of an IBSC under illumination and positive bias. $E_{\mathrm{G}}$ is the fundamental bandgap, $E_{\mathrm{L}}$ and $E_{\mathrm{H}}$ the $\mathrm{SBGs} ; \varepsilon_{\mathrm{FIB}}, \varepsilon_{\mathrm{Fe}}$. and $e_{\mathrm{Fh}}$ are the quasi-Ferni levels associated with the IB. C.B. and VB. respectively. (b) Simplified band diagram of a GaAs-capped InAs QD showing the apptoximate values of the $E_{\mathrm{L}}, E_{\mathrm{H}}$, and $E_{\mathrm{G}}$ gaps defined in the QD. IBSC model.

have to be attached on either side of the IB material to block the direct flow of carriers from the IB to the metal contacts. 1 In this arrangement, the voltage limit of the single-gap solar cell is preserved, that is, the voltage equals the split between $\varepsilon_{\mathrm{Fe}}$ and $\varepsilon_{\mathrm{Fh}}$ (divided by the electron charge). For optimal performance, another requirement is that the absorption coefficients are selective. ${ }^{17}$ This means that photons of energy higher than $E_{\mathrm{H}}$, which are susceptible to being absorbed in a VB-IB transition, should not undergo an IB-CB absorption, otherwise the thermalization losses would increase. The same applies to photons of energy higher than $E_{\mathrm{G}}$ which must not be absorbed through either of the two SBG transitions.

In the implementation with an In(Ga)As/GaAs QD array, the confined electronic ground-states of the QDs serve as the IB (Ref. 4) [see Fig. 1(b)]. The optical properties of this system can satisfy the condition of absorption selectivity defined in the IBSC model. ${ }^{18}$ In particular, photons in the energy range between $E_{\mathrm{H}}$ and $E_{\mathrm{G}}$ are highly unlikely to be absorbed in an IB-CB transition, because that would generally imply that an electron is promoted from the confined QD ground-state (wave function localized in the vicinity of the $\mathrm{QD})$ to one of the continuum states lying well above the CB minimum (delocalized wave function). ${ }^{14}$ However, all spec- tral photocurrent or quantum efficiency (QE) measurements on In(Ga)As/GaAs QD-IBSCs reported thus far $^{5-12}$ show SBG photocurrent in that spectral range.

The point to emphasize here is that the SBG photocurrent measured in QE experiments on QD-IBSCs constitutes a proof of strong IB-CB carrier escape, because by illuminating exclusively in the $E_{\mathrm{H}}-E_{\mathrm{G}}$ spectral range only the VB-IB transition is optically excited. Therefore, the observation of this photocurrent, which has been often regarded as a positive feature, is indeed indication that the QD-IBSC structure under study cannot fulfill the principle of voltage preservation. The fact that carriers can be transferred from the IB to the CB in practical QD-IBSCs without absorption of a second photon indicates that there is no positive $\left(\varepsilon_{\mathrm{Fe}}-\varepsilon_{\mathrm{FIB}}\right)$ quasi-Fermi split within the QD material, and therefore, that $e V=\varepsilon_{\mathrm{Fe}}-\varepsilon_{\mathrm{Fh}}$ cannot be maximized in spite of the use of emitters. It can be demonstrated on the base of thermodynamic arguments ${ }^{19,20}$ that the efficiency limit in the case of zero $\left(\varepsilon_{\mathrm{Fe}}-\varepsilon_{\mathrm{FIB}}\right)$ split cannot surpass the limit of single-gap cells. In fact, the extraction of SBG photocurrent from a QD-IBSC assisted by carrier escape from the IB to the CB can be related to a negative $\left(\varepsilon_{\mathrm{Fe}}-\varepsilon_{\mathrm{FIB}}\right)$ split. ${ }^{14,21,22}$ There are a number of reasons for the strength of IB-CB carrier escape/recombination in the InAs/GaAs QD system. First, the reduced value of $E_{\mathrm{L}}$. This gap is limited to about $0.2 \mathrm{eV}$ [see Fig. 1(b)] because of the confluence of an increase of the InAs gap under strain, and a reduction in the effective $E_{\mathrm{G}}$ gap $^{16}$ caused by the numerous confined hole states and the quasicontinum of states introduced by the wetting layer [(WL); characteristic of the Stranski-Krastanov growth method $\left.{ }^{23}\right]$. Also, the existence of excited QD confined states and localized energy levels associated with defects makes thermal escape easier and can assist tunneling escape processes.

\section{SAMPLE DESCRIPTION}

The strategy implemented in our samples to enlarge the IB-CB gap and reduce thermal escape is the capping of the InAs QDs with a thin InAlGaAs SRL. It has been demonstrated in the context of QD lasers and LEDs that the use of InGaAs or InAlGaAs SRLs is an effective method to redshift $E_{\mathrm{H}}{ }^{24-28}$ It has been proposed that this redshift is produced by an increase in the effective height/size of the QDs in the presence of an SRL (Refs. 25-27) and also that the reduction in the local strain in the QDs may avoid, to some extent, the blueshifting in the InAs bandgap energy. ${ }^{24,28}$ However, for the QD-IBSC application, the redshift in $E_{\mathrm{H}}$ alone is not a solution if $E_{\mathrm{L}}$ is not increased at the same time. For example, if an InGaAs SRL was applied, the SRL would form a quantum well (QW) adjacent to the dots. The $E_{\mathrm{H}}$ gap would be reduced due to strain relief but the effective value of $E_{\mathrm{L}}$ would not be as much increased, since the presence of the InGaAs QW would lower the effective CB minimum. To avoid this possibility, in the samples presented here a quaternary SRL has been implemented, where the $\operatorname{In}_{\mathrm{x}}\left(\mathrm{Al}_{\mathrm{y}} \mathrm{Ga}_{1-\mathrm{y}}\right)_{1-\mathrm{x}}$ As composition has been tuned to achieve a negligible $\mathrm{CB}$ offset with respect to $\mathrm{GaAs}^{29}$

Three samples have been fabricated, labeled SA, SB, 
TABLE I. Parameters for the three samples SA, SB, and SC analyzed in this work. Peak energy values correspond to room temperature.

\begin{tabular}{lccccccc}
\hline \hline Sample & QD layers & InGaAlAs capping & $\begin{array}{c}\text { Spacer } \\
(\mathrm{nm})\end{array}$ & $\begin{array}{c}E_{0} \\
(\mathrm{eV})\end{array}$ & $\begin{array}{c}E_{0}-E_{1} \\
(\mathrm{eV})\end{array}$ & $\begin{array}{c}\text { WL peak } \\
(\mathrm{eV})\end{array}$ & $\begin{array}{c}E_{\mathrm{A}} \\
(\mathrm{eV})\end{array}$ \\
\hline SA & 50 & No & 83 & 1.063 & 0.094 & 1.35 & 0.115 \\
SB & 30 & Yes & 84 & 0.974 & 0.101 & 1.39 & 0.224 \\
SC & $10+$ seed & Yes & 13 & 0.953 & 0.062 & $\cdots$ & $\cdots$ \\
\hline \hline
\end{tabular}

and SC. Their characteristics are compiled in Table I. Two samples contain InAlGaAs SLRs (samples SB and SC) and differ in the thickness of the spacer between the QD layers. Sample SC contains thin spacers $(13 \mathrm{~nm})$; that is in the range of the spacer thicknesses of previous QD-IBSC prototypes. ${ }^{5-11}$ Sample SB introduces the novelty of having much thicker spacers $(>80 \mathrm{~nm})$. Finally, sample SA has thick spacers and no InAlGaAs SRL. The samples were produced by molecular beam epitaxy and contain a stack of InAs/GaAs QD layers, grown in the self-assembled StranskiKrastanov mode ${ }^{23}$ and sandwiched between a Si-doped $n$-GaAs emitter (grown over a Si-doped (100) n-GaAs substrate) and an overlying Be-doped p-type GaAs emitter. Metal ohmic contacts were made by thermal evaporation and annealing using standard photolithographic methods. The QD material was $\delta$-doped with $\mathrm{Si}$ atoms to an areal density equal to the QD density, estimated from atomic force microscopy of noncapped test samples at $3-4 \times 10^{10} \mathrm{~cm}^{-2}$. The purpose of the Si $\delta$-doping is to semifill the IB states with electrons. ${ }^{4}$

Samples SA and SB contain 50 and 30 QD layers, respectively. The QDs were formed by the deposition of 2.4 monolayers (ML) of InAs at $525{ }^{\circ} \mathrm{C}$ at a rate of $0.20 \mathrm{ML} / \mathrm{s}$. A growth interrupt was introduced before the QDs were capped to promote the formation of a homogeneous population of large QDs with lower energy confined states. ${ }^{30}$ In the case of sample SB, a $2 \mathrm{~nm}$ thick InAlGaAs layer was then grown. In both cases, a thin GaAs layer ( $8 \mathrm{~nm}$ in SA, $9 \mathrm{~nm}$ in $\mathrm{SB})$ containing the $\mathrm{Si} \delta$-doping was subsequently deposited. The temperature was then raised to $5800^{\circ} \mathrm{C}$ to grow a $75 \mathrm{~nm}$ thick spacer of undoped GaAs. The increase in the temperature of the wafer eliminates residual In atoms from the growth front and smoothes the GaAs surface before the next cycle of QDs is grown. ${ }^{30}$

Sample SC contains 10 QD layers grown following the same sequence as in SB but with a slightly higher In content (2.5 ML of InAs equivalent coverage). Here the thick GaAs spacers were omitted and only the low temperature GaAs layer containing the $\mathrm{Si} \delta$-doping (13 $\mathrm{nm}$ thick in this case) was grown, although the temperature was also raised before the deposition of the next QD layer to allow surface smoothing and the evaporation of residual In. As the thin GaAs spacers in SC allow columnar correlation of the QDs, a seed layer was introduced prior to the $10 \times$ QD stack to boost the dot density. ${ }^{31,32}$ In sample SC, following two extra layers were included: a $100 \mathrm{~nm}$ thick layer of undoped GaAs was inserted between the $n$-doped emitter and the QD stack, and a $170 \mathrm{~nm}$ thick layer of light $n$-doped GaAs between the QD stack and the $p$-doped emitter. The main objective of these layers is to reduce the electric field in the dot region; they are usually referred to as field damping layers (FDLs). The motivation for including them in the QD-IBSC design has been discussed in detail elsewhere. ${ }^{33}$

Figure 2 shows transmission electron microscope (TEM) images of the three samples, taken with a JEOL 1200 EX TEM. The lack of vertical alignment between successive QD layers in samples SA and SB [Figs. 2(b) and 2(c)] indicates that the growth of thick GaAs spacers smears out and dilutes the strain-fields from individual buried QDs before the next QD layer is deposited. As a consequence, a defect-free stack containing a homogeneous QD ensemble can be grown with an increased number of layers [see Fig. 2(a) for SB; the TEM images of SA are similar]. In contrast, the residual strain concentrated over each QD site in the sample with thin GaAs spacers [SC, Fig. 2(d)] induces a columnar dot structure. Also, it can be seen that the QD ensemble in SC is not homogeneous. In Fig. 2(d) there is a trend of increasing dot size and base/height ratio with increasing number of layers and the propagation of some QD columns appears to fail in the upper layers. This indicates that the material quality in



FIG. 2. Dark field 002 TEM images: (a) low magnification image of sample SB (the whole active area can be seen, including 30 QD layers, and the emitter and window layers on top of them). (b) and (c) are high magnification images of samples SA and SB, respectively, showing two layers of dots each. (d) Active area of sample SC, where the effects of strain build-up on the growth of the upper QD layers are notable. 


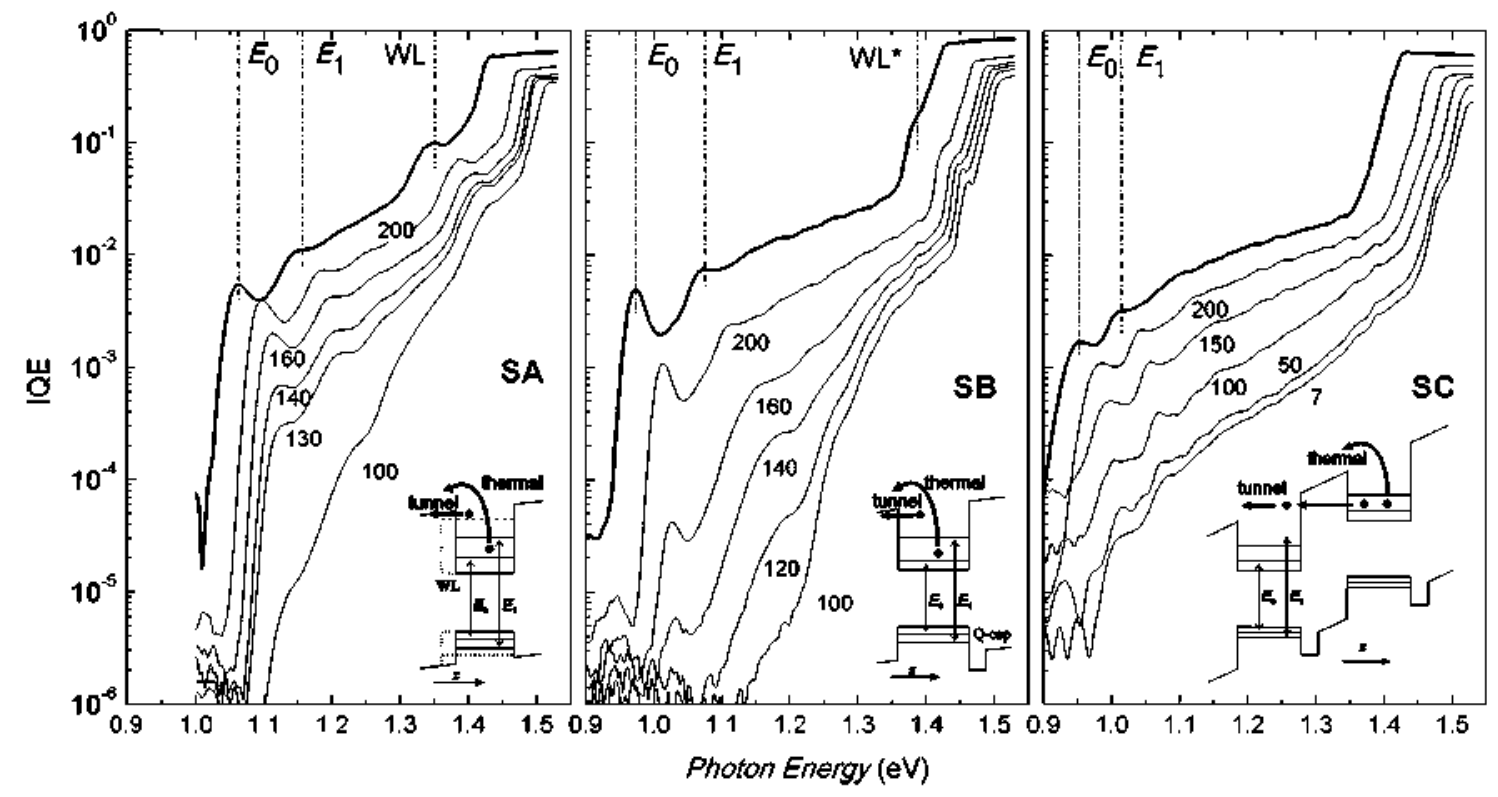

FIG. 3. IQE of samples SA, SB. and SC. The thick lines in each plot represent the measurements taken at roon tenperature and the other lines comespond each to the temperature indicated by its label (in Kelvin). The insets show a plausible QD band diagran for each sanple, as discussed in the text ( $z$ is the growth direction, dimensions are not scaled). The tunneling and thermal carriet escape mechanisms ate illustrated, as well as the transitions at energies $E_{0}$ and

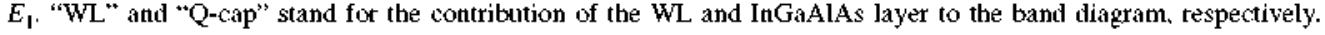

sample SC is degraded by strain build-up. In the last decade the strain build-up effect on the formation of stacked InAs nanostructures has been studied in several works ${ }^{34-36}$ and theoretically explained by anisotropic strain induced migration of atoms of the growing layer to positions above underlying nanostructures. ${ }^{37}$

\section{EXPERIMENTAL RESULTS AND DISCUSSION}

QE measurements have been carried out on the QDIBSC samples at low temperatures to evaluate the strength of thermally activated escape mechanisms and to identify potential temperature independent escape mechanisms due to tunneling. Figure 3 shows the internal quantum efficiency (IQE) versus incident photon energy of samples SA, SB, and $S C$ in the SBG spectral range. The insets illustrate simplified band diagrams and possible mechanism(s) whereby electrons escape from the QDs in each sample. The thick line in each plot corresponds to the room temperature measurement and the other curves represent data recorded at other temperatures as indicated by their respective labels. The photocurrent measurements were taken using a stabilized halogen lamp, an optical chopper, a $1 / 4 \mathrm{~m}$ grating monochromator with a resolution below $1 \mathrm{~nm}(1 \mathrm{~nm}$ at $2.8 \mu \mathrm{m})$ and low-pass filters to block photons of energy over $E_{\mathrm{G}}$. The cell was connected to a trans-impedance low-noise preamplifier that biased it at $0 \mathrm{~V}$, and the resulting photocurrent signal was demodulated using a lock-in amplifier.

The IQE measurements show a SBG photocurrent with peaks that can be attributed to different transitions between confined hole levels and confined electron levels. For simplicity, the insets represent the case where selection rules allow transitions mainly between states with the same principal quantum number $n$. That is, the first peak observed at energy $E_{0}$ has been assigned to a transition from the groundstates of holes to the ground-states of electrons, and the sec- ond peak at energy $E_{1}$ to a transition from excited hole states with $n=1$ to excited electron states with $n=1$. This is the transition configuration found theoretically ${ }^{38}$ and experimentally $^{39}$ for pyramidal InAs/GaAs QDs, although for an undetermined dot geometry the transition observed at $E_{1}$ could in principle be different, for example from the hole ground-state to the first excited electron state.

In sample SA, the ground-state transition has an energy $E_{0}=1.063 \mathrm{eV}$ at room temperature, which corresponds to the $E_{\mathrm{H}}$ gap. The difference between $E_{0}$ and $E_{1}$ is $94 \mathrm{meV}$, comparable with the largest peak energy splits found in the literature for GaAs capped InAs QDs. ${ }^{30,40}$ Compared with the $\mathrm{IQE}$ of $\mathrm{SB}$, it can be seen that the quaternary capping has a positive impact on the energy level distribution: in SB $E_{0}$ has been redshifted almost $90 \mathrm{meV}$ to $0.974 \mathrm{eV}$. Crucially, the reduction in $E_{\mathrm{H}}$ has been achieved together with an increase in the $\left(E_{0}-E_{1}\right)$ peak split, $101 \mathrm{meV}$ in $\mathrm{SB}$. The concurrence of an $E_{\mathrm{H}}$ decrease and an $\left(E_{0}-E_{1}\right)$ split increase is evidence that the SRL has served the purpose of improving the QD aspect ratio. In both cases the SBG photocurrent drops with decreasing temperature and is completely suppressed below $\sim 100 \mathrm{~K}$ for the low energy transitions. Therefore, there are no temperature independent tunneling escape mechanisms in these samples under short-circuit conditions.

It can also be observed in Fig. 3 that the decrease in the SBG photocurrent is more pronounced in SB. To estimate quantitatively the effect of the InAlGaAs SRL on thermal escape, we present in Fig. 4 an Arrhenius plot of the intensity of the ground-state transition peak. Thermally activated carrier escape is proportional to the factor $\exp \left(-E_{\mathrm{A}} / k T\right)$, where $E_{\mathrm{A}}$ is the activation energy of confined carriers, $k$ the Boltzmann constant and $T$ the temperature. In agreement with this expression, the points plotted in Fig. 4 for SA (SB) at temperatures below $\sim 170 \mathrm{~K}(\sim 200 \mathrm{~K})$ can be fitted to a straight line. From the slopes, it is deduced that $E_{\mathrm{A}}$ 


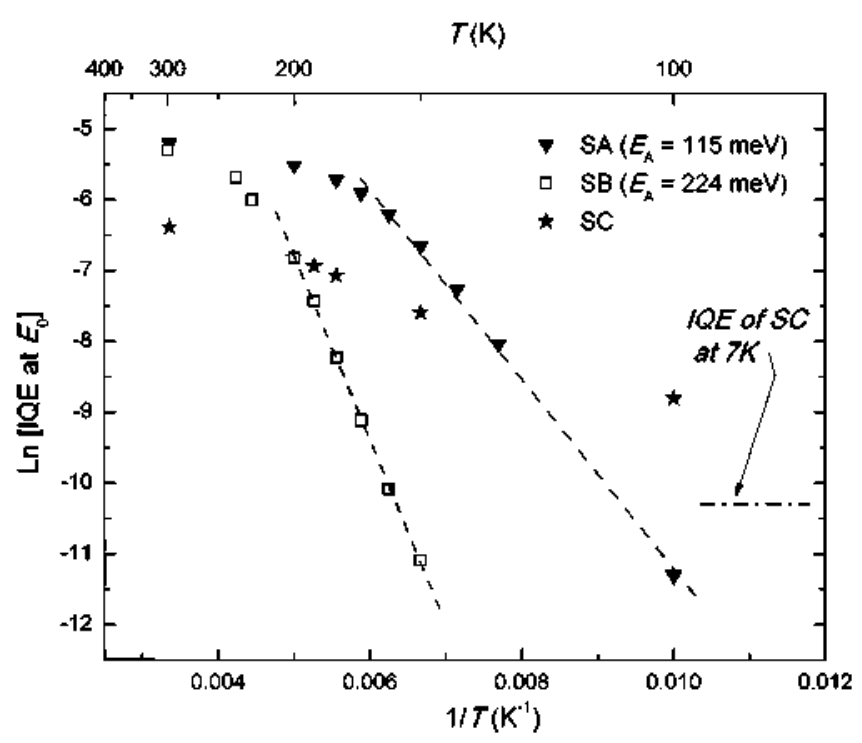

FIG. 4. An Arrhenius plot of the IQE at $E_{0}$ for samples SA, SB, and SC From the slope of the linear fit in the range whete thermal escape limits the SBG current extraction, a thermal activation energy $E_{\mathrm{A}}$ is deduced for samples $\mathrm{SA}\left(E_{\mathrm{A}}=115 \mathrm{meV}\right)$ and $\mathrm{SB}\left(E_{\mathrm{A}}=224 \mathrm{meV}\right)$.

$=115 \mathrm{meV}$ in $\mathrm{SA}$ and $224 \mathrm{meV}$ in SB. Other experimental studies have attributed an activation energy of $95 \mathrm{meV}$ to GaAs capped InAs QDs which exhibit a smaller $\left(E_{0}-E_{1}\right)$ split than sample SA. ${ }^{41}$ The increase in more than $100 \mathrm{meV}$ in the activation energy shows that using an InAlGaAs SRL is effective at reducing thermal escape in the InAs/GaAs QD-IBSC. It indicates that the reduction in $E_{\mathrm{H}}$ is not counteracted by a narrowing of $E_{\mathrm{G}}$, as would be the case if the capping layers were acting as a $\mathrm{QW}$. On the other hand, at room temperature the experimental points in the Arrhenius plot diverge from the fitted slope. This means that even in the presence of an InAlGaAs SRL, thermal escape is strong enough to allow the extraction of all the carriers generated in the QDs.

It is important to note that the difference between the activation energies of sample SA and SB measured in Fig. 4 is $109 \mathrm{meV}$, which exceeds the difference between the corresponding $E_{\mathrm{H}}$ values in Fig. $3(89 \mathrm{meV})$. To understand this fact, we have analyzed other differences between the electronic structures of both samples observed in Fig. 3. The IQE of SA has a peak at $1.35 \mathrm{eV}$ that is usually observed in QD samples and has been related to transitions between the electron and hole states introduced by the WL. In SB this peak is shifted to $1.39 \mathrm{eV}$ and is hard to distinguish at high temperatures from the GaAs bandgap edge. A similar blueshift in the $\mathrm{WL}$ in the presence of an Al-containing capping layer has been reported in Ref. 42. It was explained by the fact the total surface energy of an ultrathin $\mathrm{Al}(\mathrm{Ga}) \mathrm{As}$ layer is smaller than that of the In(Ga)As WL at the growth temperature, and therefore, In atoms in the WL are substituted by $\mathrm{Al}$ atoms during the deposition of the Al-containing cap. In the case of sample SB, the reduction in the WL confining potential is very likely partially responsible for the increase in activation energy observed. We have already mentioned that the effective $E_{\mathrm{L}}$ width in InAs/GaAs QD-IBSCs depends not only on the width of $E_{\mathrm{H}}$ but also on the energy threshold of the WL confined states [Fig. 1(b)]. This is justified by the fact that the WL states form a quasicontinum and carrier escape or relaxation between them and the barrier material bands is very fast. $^{43}$

Besides the WL signature, the IQE curves of both samples SA and SB present a collection of peaks at energies higher than $E_{1}$. At least two peaks can be seen for SA and six peaks for SB. Assuming that the transitions observed follow the same configuration between hole and electron levels, this indicates that the QDs in SB have more excited states than the QDs in SA. Photogenerated carriers in these excited states easily undergo thermal activation, and the presence of those states might also assist the thermal escape of carriers from the ground state. However, in the IQE of SB there is a difference between the behavior of the photogenerated carriers in the high energy confined states and those in the low energy states. For the high energy peaks, carrier escape is only weakly reduced by decreasing $T$, while the intensity of the $E_{0}$ and $E_{1}$ peaks is strongly suppressed. Therefore, it is concluded that in sample SB carrier escape from the ground state can be suppressed because the energy splitting between it and the low energy excited states is sufficiently large to minimize the probability of phonon scattering. Under these circumstances, the number of high energy excited states and the splitting between them is not a determining factor.

In the SBG photocurrent of sample SC (Fig. 3, right) some of the effects that we have associated with the InAlGaAs SRL are observable (notice that $E_{0}=0.953 \mathrm{eV}$ and the WL peak is not distinguishable). However, the SBG photocurrent suppression achieved in SC by reducing the temperature is much weaker than for the other two samples, and below $\sim 50 \mathrm{~K}$ it becomes almost independent of the temperature. This result indicates that a temperature independent tunneling escape mechanism is operative in sample SC under short-circuit conditions. The FDLs inserted in the structure avoid the direct tunnel of carriers from QD confined states to the emitters at the junctions but, based on our results, they are not reducing the electric field in the vicinity of the QDs enough to avoid the tunneling of carriers from the confined ground-state to the barrier material within the QD stack (see inset of Fig. 3). Given the reduced thickness of the GaAs spacers in this sample, both resonant tumneling and nonresonant tunneling are possible. ${ }^{44}$ In the first case, carriers would tunnel from the ground state of one QD to an excited state of a neighboring QD in the adjacent layer, and from there to the barrier material. The probability that the electric field allows resonant tunneling increases with the number of excited states (for sample SC at least nine peaks are observed in Fig. 3). Nonresonant tunneling can take place involving a scattering event, ${ }^{44}$ for example, involving a localized defect produced by inelastic strain relaxation.

The spacer thickness of previously fabricated QD-IBSC prototypes is typically below $20 \mathrm{~nm}$ (Refs. 5-12) and many of the designs do not include FDLs. Thus, the tunneling escape mechanism found in SC (with the contribution of direct tunneling from the QD states to the emitters if no FDLs have been implemented) can explain why the SBG photocurrent could not be reduced significantly in former low temperature experiments. ${ }^{13,45,46}$ As the elimination of carrier escape by 
tumneling is essential for preserving the voltage in QDIBSCs, a conclusion from our results is that the structure of previous QD-IBSC samples has to be revised. The thickness of GaAs spacers and the characteristics of FDLs need to be optimized for each particular design taking into account the excited QD states that can contribute to resonant tunneling under the expected voltage bias of operation. The strategy of introducing very thick spacers and no FDLs, as we have done for sample SB, is also a solution to this problem, while it also allows the growth of high quality QD layers without the need to implement strain-compensating growth methods. It has still to be determined if this strategy degrades other aspects of the QD-IBSC performance. In particular, the theoretical model presented in Ref. 47 predicts that if photon recycling effects are not important, the efficiency of a QDIBSC depends strongly on the ratio between the volume of barrier to QD material.

\section{CONCLUSIONS}

The SBG photocurrent measured in InAs/GaAs QDIBSC prototypes proves that there is a strong carrier escape between the IB and the CB. Weak carrier escape/carrier relaxation between the IB and the $\mathrm{CB}$ is the premise for a positive quasi-Fermi level split between these two bands, and therefore, for voltage preservation in the InAs/GaAs QDIBSC. We have performed QE measurements at low temperatures to identify carrier escape mechanisms in these devices. Three samples have been compared as follows: in sample SA conventional InAs/GaAs QD layers were separated by thick ( $84 \mathrm{~nm}$ ) GaAs spacers, in sample $S B$ InAlGaAs capped QD layers were also separated by thick GaAs spacers and in sample SC InAlGaAs capped QD layers were separated by thin $(13 \mathrm{~nm})$ GaAs spacers, which is in the range of spacer thickness of previous QD-IBSCs. From them, the structure of sample SB has proved to be the most appropriate with respect to the strength of IB-CB carrier escape.

The samples with thick spacers exhibit only thermally activated carrier escape from the ground-states of the QDs, whereas in SC a tunneling escape mechanism is also present. To block tunnel escape and pursue voltage preservation in future InAs/GaAs QD-IBSCs it will be required to optimize FDLs and GaAs spacer thicknesses, taking into account particular characteristics of the sample such as the number of excited QD states that can assist resonant tunneling.

By comparing samples SA and SB, where tunneling escape could be fully eliminated under short-circuit conditions, it is concluded that the introduction of a $2 \mathrm{~nm}$ thick InAlGaAs SRL improves the QD confined states energy distribution and reduces the confining potential of the WL. As a result, thermal escape is significantly reduced, enhancing the activation energy of confined carriers in the QD groundstates from 115 to $224 \mathrm{meV}$.

\section{ACKNOWLEDGMENTS}

This work has been supported by the IBPOWER project funded by the European Commission (Grant No. 211640), by the Regional Government of Madrid within the project NU-
MANCIA2 (Grant No. S2009/ENE-1477), and by the Spanish National Research Program within the project GENESIS-FV (Grant No. CSD2006-0004). T.B., A.M.S., and S.I.M. acknowledge support from MCI (Grant No. TEC2008-06756-C03-(02/TEC) and Andalusian Regional Government (Grant No. P08-TEP-03516).

'A. Lugue and A. Martí, Phys. Rev. Lett. 78, 5014 (1997).

${ }^{2}$ W. Shockley and H. J. Queisser. J. Appl. Phys. 32, 510 (1961).

${ }^{3}$ G. L. Araujo and A. Martí, Sol. Energy Mater. Sol, Cells 33, 213 (1994).

${ }^{4} \mathrm{~A}$. Marti. L. Cuadra, and A. Luque, Conference Record of the 28th IEEE Photovoltaics Specialists Conference (IEEE, New York, USA, 2000), pp. 940-943.

${ }^{5}$ A. Martí. N. López. E. Antolín. E. Cánovas. C. Stanley. C. Farmer, L. Cuadra, and A. Luque, Thin Solid Films 511-512, 638 (2006).

${ }^{6}$ S. M. Hubbard, C. D. Cress, C. G. Bailey, R. P. Raffaelle, S. G. Bailey, and D. M. Wilt, Appl. Phys. Lett. 92, 123512 (2008).

${ }^{7}$ R. B. Laghumavarapu, M. El-Emawy, N. Nuntawong. A. Moscho, L. F. Lester, and D. L. Huffaker, Appl. Phys. Lett. 91, 243115 (2007).

${ }^{8}$ R. Oshima, A. Takata, and Y. Okada, Appl. Phys. Lett. 93, 083111 (2008).

${ }^{9}$ V. Popescu. G. Bester, M. C. Hanna. A. G. Norman, and A. Zunger, Phys. Rev. B 78, 205321 (2008).

${ }^{10}$ S. A. Blokhin, A. V. Sakharov, A. M. Nadtochy, A. S. Pauysov, M. V. Maximov, N. N. Ledentsov, A. R. Kovsh. S. S. Mikhtin, V. M. Lantratov, S. A. Mintairov. N. A. Kaluzhniy, and M. Z. Shvarts. Semiconductors 43. $514(2009)$.

${ }^{\prime \prime}$ S. M. Hubbard, C. G. Bailey, C. D. Cress, S. Polly, J. Clark, D. V. Forbes, R. P. Raffaelle. S. G. Bailey, and D. M. Wilt. Conference Record of the 33rd IEEE Photovoltaic Specialists Conference (IEEE. San Diego, USA. 2008).

${ }^{12}$ A. Luque, A. Martí, N. López, E. Antolín, E. Cánovas, C. Stanley, C. Farmer. L. J. Caballero, L. Cuadra, and J. L. Balenzategui, Appl. Phys. Lett. 87. 083505 (2005).

${ }^{13}$ A. Martí, E. Antolín, C. R. Stanley, C. D. Farmer. N. López, P. Díaz, E. Canovas, P. G. Linates, and A. Luque, Phys. Rev. Lett. 97. 247701 (2006).

${ }^{14}$ A. Martí, C. R. Stanley, and A. Luque. in Nanostructured Materials for Solar Energy Conversion. edited by T. Soga (Elsevier Science, Amsterdam. 2006), pp. 539-566.

${ }^{15}$ A. Luijue and A. Martí, Prog. Photovolt. Res. Appl. 9, 73 (2001).

${ }^{16}$ A. Martí, L. Cuadra, and A. Luque. in Next Generation Photovoltaics: High Efficiency through Full Spectrum Utilization, edited by A. Martí and A. Lugue (Institute of Physics. Bristol, 2003), pp. 140-162.

${ }^{17}$ L. Cuadta, A. Martí, and A. Luque, IEEE Trans. Electron Devices 51, $1002(2004)$

${ }^{18}$ A. Martí, E. Antolín. E. Cánovas. N. López. A. Luque. C. R. Stanley. C. D. Farmer, P. Diaz, C. Christofides, and M. Buthan. Proceedings of the 21st European Photovoltaic Solar Energy Conference (WIP-Renewable Energies. Dresden. Germany, 2006). pp. 99-102.

${ }^{19}$ A. Luque and A. Martí. Phys. Rev. B 55, 6994 (1997).

${ }^{20} \mathrm{~A}$. Luque, A. Martí, and L. Cuatra, IEEE Trans. Electron Devices 48, $2118(2001)$.

${ }^{21}$ A. Luque, A. Martí, C. Stanley. N. López, L. Cuadra. D. Zhou, and A. Mc-Kee, J. Appl. Phys. 96.903 (2004).

${ }^{22}$ E. Antolín, A. Martí, P. G. Linares, E. Cánovas, D. Fuertes-Martón, A. Luque, C. D. Farmer, and C. R. Stanley, Proceedings of the $23 \mathrm{rd}$ European Photovoltaic Solar Energy Conference (WIP-Renewable Energies. Valencia, Spain. 2008), pp. 5-10.

${ }^{23}$ I. N. Sttanski and L. V. Krastanov, Akad. Wiss. Lit. Mainz Abh. Math. Naturwiss. Kl. 146, 797 (1939).

${ }^{24}$ V. M. Ustinov, N. A. Maleev, A. E. Zhukov, A. R. Kovsh, A. Y. Egorov, A. V. Lunev, B. V. Volovik. I. L. Krestnikov, Y. G. Musikhin, N. A. Bert. P. S. Kop 'ev, and Z. I. Alferov, Appl. Phys. Lett. 74, 2815 (1999).

${ }^{25}$ V. M. Ustinov, A. E. Zhukov, A. R. Kovsh, N. A. Maleev, S. S. Mikhrin, A. F. Tsatsul' nikov, M. V. Maximov. B. V. Volovik, D. A. Bedarev. P. S. Kop'ev. Z. I. Alferov, L. E. Vorob'ev. D. A. Firsov, A. A. Suvorova, I. P. Soshnikov, P. Werner, N. N. Ledentsov, and D. Bimbery, Microelectron. J. 31. $1(2000)$.

${ }^{26}$ M. V. Maximov, A. F. Tsatsul'nikov, B. V. Volovik. D. S. Sizov, Y. M. Shernyakov, I. N. Kaiander. A. E. Zhukov, A. R. Kovsh. S. S. Mikhrin. V. M. Ustinov, Z. I. Alferov, R. Heitz, V. A. Shchukin, N. N. Ledentsov, D. Bimberg, Y. G. Musikhin, and W. Neumann, Phys. Rev. B 62, 16671 (2000).

${ }^{27}$ K. Nishi. H. Saito. S. Sugou, and L. Jeong-Sik. Appl. Phys. Lett. 74, 1111 
(1999).

${ }^{28}$ J. Tatebayashi. M. Nishioka, and Y. Arakawa, Appl. Phys. Lett. 78, 3469 (2001).

${ }^{29}$ P. G. Linares, C. D. Farmer, E. Antolín. S. Chakrabartib. A. M. Sánchez. T. Ben, S. I. Molina, C. R. Stanley, A. Martí, and A. Luque, "InGaAlAs quaternary alloys for quantum dot intermediate band solar cells," Energy Procedia (to be published).

${ }^{30}$ S. Fafard. Z. R. Wasilewski, C. N. Allen, D. Picard. M. Spanner, J. P. McCaffrey, and P. G. Piva, Phys. Rev. B 59, 15368 (1999).

${ }^{31}$ P. Howe, E. C. L. Ru, E. Clarke, B. Abbey, R. Murtay, and T. S. Jones, J. Appl. Phys. 95. 2998 (2004).

${ }^{32}$ P. Howe. E. C. L. Ru, E. Clarke. R. Muiray, and T. S. Jones, J. Appl. Phys. 98, $113511(2005)$

${ }^{33}$ A. Martí, E. Antolín, E. Cánovas, N. López, P. G. Linares, A. Luque, C. R. Stanley, and C. D. Farmer. Thin Solid Films 516, 6716 (2008).

${ }^{34}$ S. I. Molina, T. Ben. D. L. Sales, J. Pizarto, P. L. Galindo. M. Varela, S. J. Pennycook, D. Fuster, Y. González, and L. González, Nanotechnology 17, $5652(2006)$.

${ }^{35}$ S. I. Molina, M. Varela, T. Ben, D. L. Sales, J. Pizarto, P. L. Galindo, D. Fuster. Y. González, L. González, and S. J. Pennycook, J. Nanosci. Nanotechnol. 8, 3422 (2008).

${ }^{36} \mathrm{M}$. Gutiértez, M. Herrera, D. González, R. García, and M. Hopkinson, Appl. Phys. Lett. 88, 193118 (2006).
${ }^{37}$ Q. Xie. A. Madhukar, P. Chen, and N. P. Kobayashi, Phys. Rev. Lett. 75, 2542 (1995).

${ }^{38}$ M. A. Cusack. P. R. Briddon, and M. Jaros, Phys. Rev. B 56. 4047 (1997).

${ }^{39}$ I. E. Itskevich, M. S. Skolnick. D. J. Mowbray, I. A. Trojan. S. G. Lyapin. L. R. Wilson, M. J. Steet, M. Hopkinson, L. Eaves, and P. C. Main, Phys. Rev. B 60, R2185 (1999).

${ }^{40}$ O. B. Shchekin, G. Park, D. L. Huffaker. and D. G. Deppe. Appl. Phys. Lett. 77. $466(2000)$.

${ }^{4 \mid}$ P. W. Fry, I. E. Itskevich, S. R. Parnell, J. J. Finley, L. R. Wilson, K. L. Schumacher. D. J. Mowbray, M. S. Skolnick, M. AJ-Khafaji, A. G. Cullis, M. Hopkinson, J. C. Clark. and G. Hill. Phys. Rev. B 62, 16784 (2000).

${ }^{42}$ A. F. Tsatsul'nikov, A. R. Kovsh. A. E. Zhukov, Y. M. Shernyakov, Y. G. Musikhin, V. M. Ustinov, N. A. Bett, P. S. Kop'ev, Z. I. Alfetov, A. M. Mintairov, J. L. Merz, N. N. Ledentsov, and D. Bimberg, J. Appl. Phys. $\mathbf{8 8}, 6272(2000)$

43. J. Nozik, Annu. Rev. Phys. Chem. 52, 193 (2001).

${ }^{44}$ A. M. Fox, R. G. Ispasoin. C. T. Foxon, J. E. Cunningham, and W. Y. Jan, Appl. Phys. Lett. 63, 2917 (1993).

${ }^{45}$ E. Antolín, A. Martí. C. R. Stanley. C. D. Farmer, E. Cánovas, N. López. P. G. Linares, and A. Luque. Thin Solid Films 516. 6919 (2008).

${ }^{46}$ C. D. Cress, S. M. Hubbard, C. Bailey, R. Robinson, B. J. Landi, and R. P. Raffaelle, MRS Symposia Proceedings 1031. H13-19 2008).

${ }^{47}$ A. Martí, L. Cuadra, and A. Luque, Physica E 14. 150 (2002). 